\title{
Recherches sur la résistance des sols aux mala- dies. XIV. Modification du niveau de récepti- vité d'un sol résistant et d'un sol sensible aux fusarioses vasculaires en réponse à des apports de fer ou de glucose
}

Philippe LEMANCEAU, Claude AlabouvetTE $\left({ }^{*}\right)$ \& Yvonne COUTEAUdiER $\left({ }^{*}\right)$

E.N.I.T.H., 2, rue Le Nôtre, F 49045 Angers Cedex

(*) I.N.R.A., Station de Recherches sur la Flore pathogène dans le Sol, 17, rue Sully, F 21024 Dijon Cedex

La résistance aux fusarioses vasculaires des sols de Salinas Valley est attribuée à la compétition pour le fer qui limite le développement des Fusarium. Les sols de Châteaurenard et de Salinas Valley ayant en commun leur faible concentration en fer facilemenı extractible, le but de cet article esı d'évaluer le rôle de la compétition pour le fer dans les mécanismes de résistance des sols de Châteaurenard.

L'apport de fer chélaté à EDTA, utilisable pour les Fusarium, se traduit par une augmentation de la gravité de la maladie. Au contraire, l'apport du ligand EDDHA, présentant une forte affinité pour le fer, provoque une diminution de la concentration en fer disponible et corrélativement une diminution de la gravité de la maladie. Des résultats similaires ont été enregistrés dans un sol sensible à la maladie indiquant que la compétition pour le fer conditionne en partie le niveau de réceptivité des sols aux fusarioses vasculaires.

Parallèlement l'enrichissement des sols avec le glucose provoque une augmentation de la gravité de la maladie indiquant que la compétition pour l'énergie conditionne également le niveau de réceptivité des sols aux fusarioses.

L'augmentation de la gravité de la maladie à la suite d'apports simultanés de fer et de glucose indique que les 2 mécanismes de compétition s'expriment conjointement dans les mêmes sols.

Mots clés additionnels : Compétition nutritive, ligands, chélates de fer, EDTA, EDDHA, FeEDTA, FeED. DHA.

Studies on the disease suppressiveness of soils. XIV. Modification of the receptivity level of a suppressive and a conducive soil to fusarium wilt in response to the supply of iron or of glucose.

The fusarium wilt suppressiveness of the Salinas Valley soils is attributed to competition for iron which limits Fusarium development. The Châteaurenard and Salinas Valley soils having in common a low content of easily extractable iron, the aim of this research was to evaluate the role of iron competition in the suppressiveness mechanism of Châteaurenard soils. If EDTA-chelated iron, available to Fusarium, was supplied, this led to an increase in disease incidence. In contrast, when the ligand EDDHA (showing a high affinity for iron) was supplied there was a decrease in available iron content and correlatively a decrease in disease incidence. Similar results were recorded with a conducive soil, indicating that the receptivity level of the soils to fusarium wilt depends in part on competition for iron. In the same way, soil enrichment with glucose induced an increase in disease incidence, indicating that the receptivity level of the soils to fusarium wilt also depends on competition for energy. The increased disease incidence which followed simultaneous supply of iron and glucose indicated that the two mechanisms of competition can both be expressed in the same soils.

Additional key words : Competition for nutrients, iron chelates, ligands, EDTA, EDDHA, FeEDTA, FeEDDHA. 


\section{INTRODUCTION}

Mis en évidence dès la fin du siècle dernier (ATKINSON, 1892) le phénomène de résistance des sols aux fusarioses vasculaires a été largement étudié (TousSOUN, 1975). Deux modèles ont été l'objet, au cours des 10 dernières années, de recherches les plus approfondies. Ce sont les sols de Salinas Valley (Californie) (SCHER \& BAKER, 1980 ; BAKER et al., 1986) et de Châteaurenard (France) (LouvET et al., 1976 ; ALABOUVETTE, 1986). Les mécanismes de cette résistance, de nature microbiologique, sont encore mal connus et 2 théories principales ont été avancées. Ainsi RouXEL et al. (1979) démontrent la participation des Fusarium non pathogènes à la résistance des sols de Châteaurenard. AlabouvetTe (1983) attribue ensuite à la compétition pour l'énergie, liée à l'importance et à l'activité de la biomasse microbienne, un rôle important dans les mécanismes de résistance et, plus généralement, dans les interactions microbiennes qui conditionnent le niveau de réceptivité des sols aux fusarioses vasculaires. SCHER \& BAKER $(1980,1982)$ démontrent que la compétition pour le fer, qui s'exerce dans les sols de Salinas entre les populations de Pseudomonas fluorescents et le Fusarium pathogène, joue un rôle primordial dans ces mécanismes de résistance. En effet, dans les sols à pH élevé $(>6,5)$ la concentration en fer ferrique (FeIII) assimilable par les microorganismes est extrêmement faible. Les microorganismes aérobies font alors appel à un système actif d'acquisition de FeIII, basé sur la production de sidérophores et de protéines réceptrices membranaires (NEILANDS, 1973 ; LEONG, 1986). Les sidérophores microbiens ne présentent pas tous la même affinité pour le FellI. Ainsi, les pyoverdines produites par les Pseudomonas (MEYER \& ABDALLAH, 1978) forment avec le FeIII des complexes beaucoup plus stables que les fusarinines synthétisées par les Fusarium (EMERY, 1965). Le fer chélaté par les pyoverdines est alors indisponible pour les Fusarium; la croissance des tubes germinatifs issus de leurs chlamydospores est réduite (SCHER \& BAKER, 1982), ce qui diminue d'autant la probabilité d'infecter la racine des plantes.

Pour mettre en évidence la relation qui existe entre la disponibilité en fer et le niveau de résistance des sols de Salinas, SCHER \& BAKER (1982) ont utilisé des chélates organiques de synthèse présentant une constante de stabilité supérieure (FeEDDHA) ou inférieure (FeEDTA) à celle du complexe Fer-fusarinine.

De manière similaire, c'est en apportant au sol des concentrations croissantes de glucose qu'ALABOUVETTE et al., (1985a) ont démontré que le niveau de réceptivité des sols est en relation avec leur niveau énergétique. Le carbone est un élément indispensable à la germination de chlamydospores de Fusarium soumises à la fongistase des sols (AlABOUVETTE et al., 1985a ; LOCKWOOD, 1977).

Outre leur résistance aux fusarioses vasculaires, les sols de Salinas et de Châteaurenard ont en commun plusieurs caractéristiques physico-chimiques : $\mathrm{pH}$ et teneur en calcaire total élevés, faible concentration en fer facilement extractible. Cette similitude nous a incités à reproduire avec le sol de Châteaurenard les expérimentations de SCHER \& BAKER (1982) el à comparer, dans un même sol, l'effet de l'enrichissement en glucose ou en fer sur le niveau de réceptivité aux fusarioses. Il est intéressant de savoir si les 2 types de mécanismes mis en évidence séparément peuvent se manifester simultanément dans un même sol et de déterminer s'ils sont en relation avec les caractéristiques physico-chimiques des sols et leur résistance aux fusarioses vasculaires. Le $\mathrm{pH}$ est en effet un des facteurs principaux qui détermine la quantité de FeIII assimilable par les microorganismes. Pour cette raison, les expérimentations ont été réalisées parallèlement avec le sol résistant de Châteaurenard de $\mathrm{pH}$ élevé et avec un sol sensible de pH plus faible.

\section{MATÉRIEL ET MÉTHODES}

\section{A. Les sols}

Tous les essais sont réalisés avec un seul et même prélèvement de chaque sol dont les caractéristiques sont présentées dans le tableau 1.

Le sol résistant aux fusarioses vasculaires provient de Châteaurenard (Bouches-du-Rhône), le sol sensible de Carquefou (Loire-Atlantique). La texture du sol de Châteaurenard est limono-argileuse, celle du sol de Carquefou sablo-limoneuse. Contrairement au sol sensible, le $\mathrm{pH}$ et la teneur en calcaire total du sol résistant sont élevés. Ces 2 caractéristiques expliquent la très faible concentration en fer facilement extractible du sol résistant, 15 fois inférieure à celle du sol sensible. La concentration en fer amorphe est par ailleurs analogue dans les 2 sols.

\section{B. Mesure de la réceptivité}

La réceptivité des sols aux fusarioses vasculaires est mesurée en suivant la méthode standardisée établie par AlabouvetTE et al. (1982). Selon les essais, les sols sont infestés avec une ou plusieurs concentrations d'inoculum, sous forme de propagules conservées dans le talc (Tello-Marquina et al., 1980). Pour chaque traitement expérimental, le sol est réparti en 6 pots de $500 \mathrm{ml}$ dans lesquels sont semées 10 graines d'une variété de lin ( Hera »), sensible à la maladie. Ces plantes sont cultivées pendant 9 semaines en chambre climatisée (photopériode 16 heures, température de jour $25^{\circ} \mathrm{C}$, température de nuit $20^{\circ} \mathrm{C}$, humidité relative $60 \mathrm{p}, 100$ ). Chaque semaine le nombre de plantes malades est noté.

\section{Modification de la disponibilité en fer et en car- bone des sols}

La disponibilité en fer des sols est modifiée par apport de ligands EDDHA (acide éthylène diamine $\mathrm{O}$ hydroxyphénylacétique), EDTA (acide éthylène diamine tétracétique) ou de leurs chélates FeEDDHA, FeEDTA. La dissolution de ces produits est réalisée selon la technique décrite par SCHER \& BAKER (1982). Selon les essais, des concentrations croissantes de ligand chélaté ou non $(100,300$ et $500 \mu \mathrm{g} / \mathrm{g}$ sol sec) sont apportées aux sols. 
TABLEAU 1

Caractéristiques physico-chimiques et agronomiques des sols de Châteaurenard et Carquefou.

Physico-chemical and agronomical properties of soils from Châteaurenard and Carquefou.

\begin{tabular}{|c|c|c|c|c|}
\hline Granulométrie & & & Châteaurenard & Carquefou \\
\hline Argile & & p. 1000 & 227 & 50 \\
\hline Limon fin & & p. 1000 & 152 & 78 \\
\hline Limon grossier & & p. 1000 & 192 & 84 \\
\hline Sables fins & & p. 1000 & 98 & 24 \\
\hline Sables grossiers & & p. 1000 & 5 & 764 \\
\hline $\mathrm{pH}$ eau & & & 7,9 & 5,3 \\
\hline Carbone organique (méthode Anne) & & p. 1000 & 22,6 & 18,04 \\
\hline Matières organiques & & p. 1000 & 38,8 & 31,02 \\
\hline Azote Kjeldahl & & & 2,93 & 1,42 \\
\hline $\mathrm{C} / \mathrm{N}$ & & p. 1000 & 7,71 & 12,70 \\
\hline Calcaire total & & p. 1000 & 318 & 0 \\
\hline Acide phosphorique (Joret Hebert) & & p. 1000 & 0,29 & 0,555 \\
\hline Capacité d'échange (méthode Metson) & m.eq. & p. 100 & 12,7 & 7,03 \\
\hline Calcium échangeable & m.eq. & p. 100 & 37,4 & 8,44 \\
\hline Magnésium échangeable & m. eq. & p. 100 & 1,96 & 1,84 \\
\hline Potassium échangeable & m. eq. & p. 100 & 0,915 & 1,54 \\
\hline Manganèse échangeable & & p.p.m. & 3,3 & 1,4 \\
\hline Fer facilement extractible (méthode Juste \& Pouget) & & p.p.m. & 18,8 & 279,9 \\
\hline Fer amorphe (méthode Jeanroy) & & p. 100 & 0,11 & 0,09 \\
\hline Calcium total & & p. 100 & 13,13 & 0,51 \\
\hline Magnésium total & & p. 100 & 0,8 & 0,209 \\
\hline Potassium total & & p. 100 & 0,96 & 2,435 \\
\hline Sodium total & & p. 100 & 0,65 & 0,36 \\
\hline
\end{tabular}

La teneur des sols en carbone organique facilement assimilable est augmentée par l'apport de glucose en solution. Selon les essais, les concentrations croissantes utilisées sont 0,$1 ; 0,5 ; 1 ; 5 \mathrm{mg} / \mathrm{g}$ sol sec.

Tous les amendements sont fractionnés en 3 apports : le premier lors du semis, les 2 suivants, 1 et 2 semaines plus tard.

\section{RÉSULTATS}

\section{A. Comparaison du niveau de réceptivité des sols étudiés}

Les pourcentages de plantes malades observées dans les sols de Châteaurenard et de Carquefou infestés avec Fusarium oxysporum f. sp. lini aux doses de 5000 et 10000 germes/g sol (fig. 1) confirment le bon niveau de résistance aux fusarioses vasculaires du sol de Châteaurenard, et la sensibilité du sol de Carquefou.

Les densités d'inoculum de 5000 germes/g pour le sol sensible et 10000 germes $/ g$ pour le sol résistant ont été retenues lors des essais ultérieurs car elles permettent de bien apprécier les variations du niveau de réceptivité des sols liés à divers traitements expérimentaux.

\section{B. Modifications de la réceptivité des sols aux fusa- rioses vasculaires par apport de ligands et chélates de fer}

Le premier essai (fig. 2) reprend en partie le protocole établi par SCHER \& BAKER (1982). EDDHA et FeEDTA sont apportés aux sols à 2 concentrations (100 et $300 \mu \mathrm{g} / \mathrm{g}$ ).

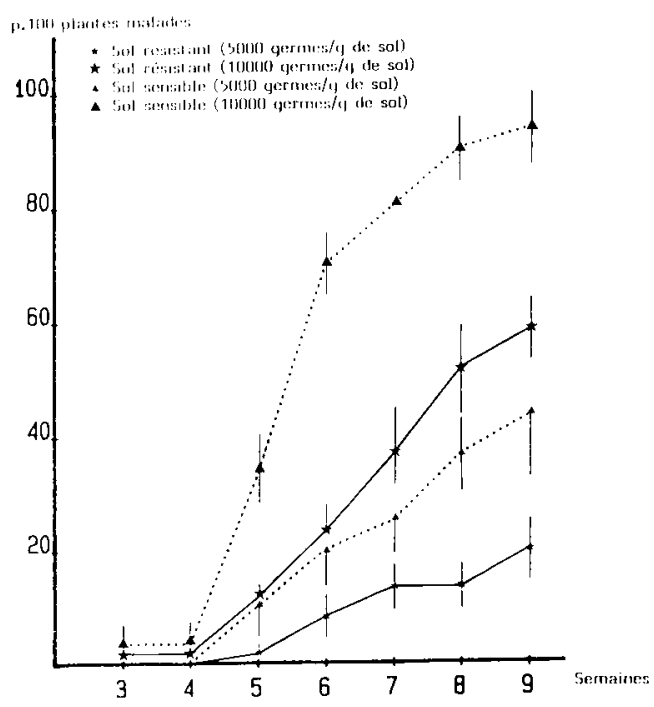

Figure 1

Comparaison de la réceptivité des sols résistant (Châteaurenard) et sensible (Carquefou) à la fusariose vasculaire du lin : pourcentage de plantes malades après infestation des sols avec F. o. f. sp. lini à la dose de 5000 et 10000 germes $/ g$ de sol.

Moyennes de 6 répétitions de 10 plantes suivies de leurs écarts types. Comparison of the receptivity to fusarium wilt of flax of the suppressive (Châteaurenard) and conducive (Carquefou) soils : percentage of wilted plants after soil infestation at 5000 and 10000 C.F.U./g of soil.

Means of 6 replications of 10 plants followed by their standard deviations.

Pour chaque sol, la gravité de la maladie est réduite par apport du ligand EDDHA et ce d'autant plus que sa concentration est élevée. A l'opposé la gravité est significativement augmentée par les amendements en chélate FeEDTA, à la concentration de $300 \mu \mathrm{g} / \mathrm{g}$. 


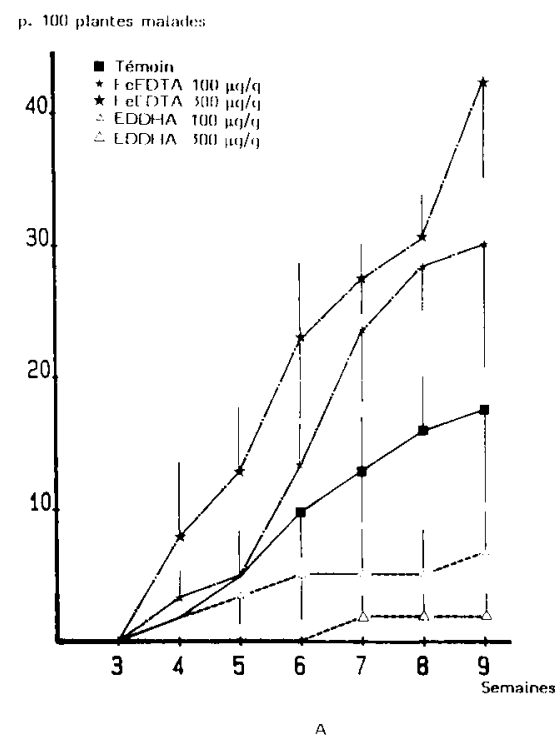

Figure 2

Réceptivité des sols résistant (A) et sensible (B) amendés avec EDDHA ou FeEDTA ( 100 et $300 \mu \mathrm{g} / \mathrm{g}$ ) à la fusariose vasculaire du lin : pourcentage de plantes malades après infestation des sols avec F. o. f. sp. lini à la dose $10000(A)$ ou $5000(B)$ germes $/ g$ de sol. Moyennes de 6 répétitions de 10 plantes suivies de leurs écarts types.

Dans le second essai (fig. 3), l'effet de chaque ligand est comparé à celui de son chélate afin de mieux apprécier le rôle du fer (FeIII). Chaque élément est apporté à 3 concentrations : 100,300 et $500 \mu \mathrm{g} / \mathrm{g}$.

A concentration égale, les augmentations de la gravité de la maladie induites par le ligand EDTA ou son chélate FeEDTA sont peu différentes (fig. 3). La concentration minimale suffisante pour élever le niveau de réceptivité est $100 \mu \mathrm{g} / \mathrm{g}$ pour le sol sensible et $300 \mu \mathrm{g} / \mathrm{g}$ pour le sol résistant. La légère différence de mortalité, entre les traitements 300 et $500 \mu \mathrm{g} / \mathrm{g}$, observée lors des premières semaines disparaît ensuite.

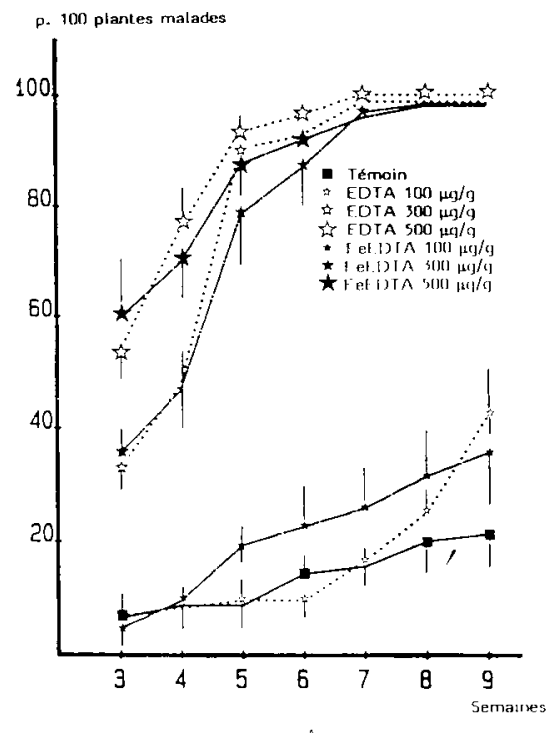

Figure 3

Réceptivité des sols résistant (A) et sensible (B) amendés avec EDTA ou FeEDTA, EDDHA ou FeEDDHA (100 et 300 et 500 $\mu \mathrm{g} / \mathrm{g}$ ) à la fusariose vasculaire du lin : pourcentage de plantes malades après infestation du sol avec F. o. $f$. sp. lini aux doses 10000 (A) ou 5000 (B) germes/g de sol.

Moyennes de 6 répétitions de 10 plantes suivies de leurs écarts types.

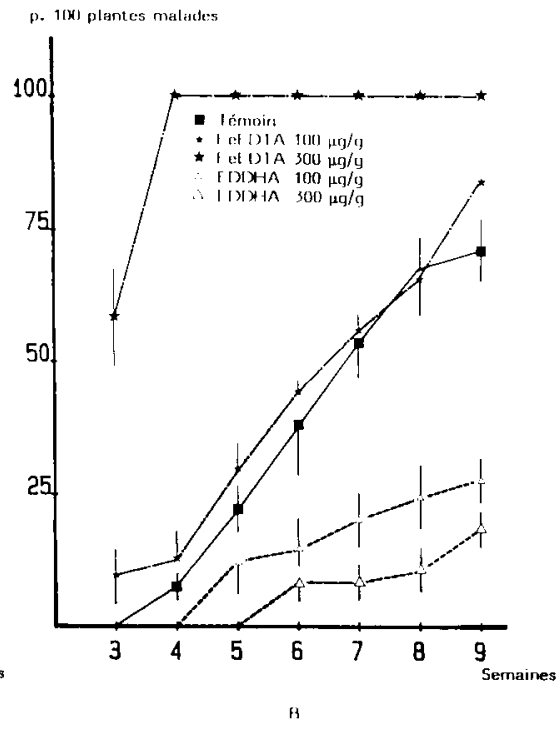

Receptivity to fusarium will of flax of the suppressive (A) and con ducive (B) soils supplemented with EDDHA or FeEDTA 1100 and $300 \mu \mathrm{g} / \mathrm{g})$ : percentage of wilted plants after soil infestation with F. o.f. sp. lini at 10000 (A) or 5000 (B) C.F.U. $/ \mathrm{g}$ of soil.

Means of 6 replications of 10 plants followed by their standard deviations.

L'induction de résistance assurée par l'apport de FeEDDHA, d'autant plus nette que la concentration est élevée, est supérieure à celle obtenue avec EDDHA. La dose la plus forte de ce chélate $(500 \mu \mathrm{g} / \mathrm{g})$ est celle qui permet d'assurer la meilleure protection contre la fusariose vasculaire (fig. 3).

Ces résultats doivent cependant être nuancés, car les plantes cultivées dans les sols amendés avec EDTA présentent des signes de phytotoxicité se manifestant par un jaunissement accusé des plantes et une faible croissance. Des cultures de lin ont été réalisées dans les sols amendés, mais sans infestation par l'agent

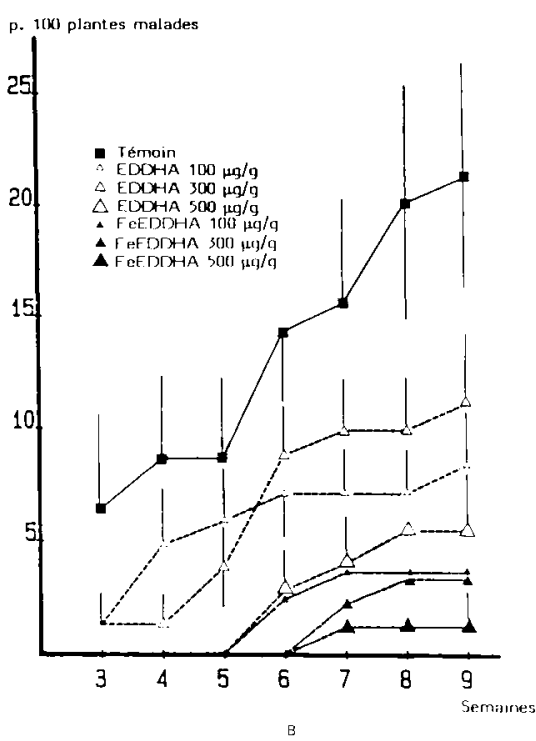

Receptivity to fusarium wilt of flax of the suppressive (A) and conducive (B) soils supplemented with EDDHA, FeEDTA, EDDHA or FeEDDHA (100, 300 and $500 \mu \mathrm{g} / \mathrm{g}):$ percentage of wilted plants after soil infestation with F. o. f. sp. lini at $10000(A)$ or $5000(B)$ C.F.U./g of soil.

Means of 6 replications of 10 plants followed by their standard deviations. 
pathogène. Les résultats montrent que EDTA et FeEDTA à $100 \mu \mathrm{g} / \mathrm{g}$ provoquent la mort de $100 \mathrm{p} .100$ et 89 p. 100 des plantes dans le sol de Carquefou. A cette dose, la phytotoxicité est très faible dans le sol de Châteaurenard; la mortalité limitée à 3 p. 100 après apport du ligand EDTA est nulle avec le chélate FeEDTA. Par contre, EDDHA et FeEDDHA ne sont responsables d'aucune phytotoxicité apparente.

\section{Modification de la réceptivité aux fusarioses vascu- laires des sols par apport de glucose}

Les sols infestés avec $F$. $O$. f. sp. lini sont respectivement amendés avec les concentrations de glucose suivantes : 0,$1 ; 0,5 ; 1 \mathrm{mg} / \mathrm{g}$ pour Carquefou, 0,1 ; 0,$5 ; 1 ; 5 \mathrm{mg} / \mathrm{g}$ pour Châteaurenard.

Globalement, l'enrichissement des sols en glucose détermine une augmentation de la gravité de la maladie (fig. 4). Cet effet dépend à la fois de la concentration et du sol dans lequel le glucose est introduit. Ainsi pour le sol sensible (fig. 4B), cette élévation est proportionnelle à la concentration employée $(0,1$; 0,$5 ; 1 \mathrm{mg} / \mathrm{g}$ ). Par contre, ces concentrations sont insuffisantes pour induire une modification sensible de la réceptivité du sol de Châteaurenard (fig. 4A). Seul l'apport de $5 \mathrm{mg}$ de glucose/g de sol détermine une augmentation sensible de la mortalité des plantes.
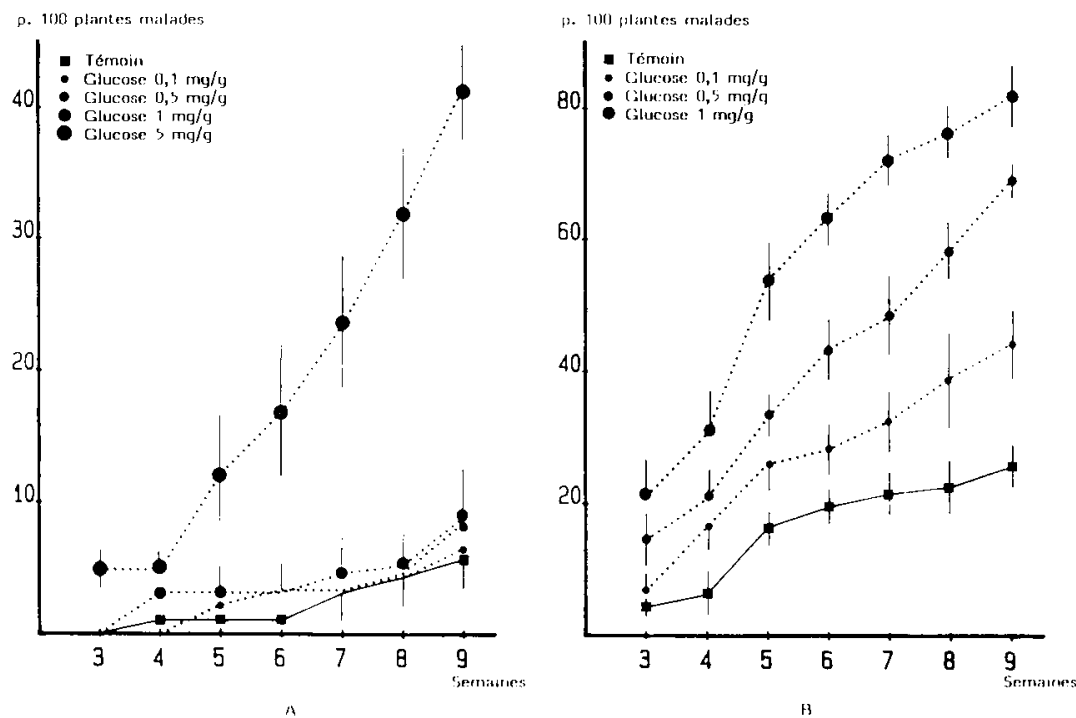

Figure 4

Receptivity to fusarium wilt of flax of the suppressive (A) and conducive (B) soils supplemented with increasing levels of glucose : percentage of wilted plants after soil infestation with $\mathrm{F}$. o. $f$. sp. lini at 10000 (A) or 5000 (B) C.F.U./g of soil.

Means of 6 replications of 10 plants followed by their standard deviations.

Receptivite des sols résistant (A) et sensible (B) amendés avec concentrations croissantes de glucose à la fusariose vasculaire du lin : pourcentage de plantes malades après infestation du sol avec F. o.f. sp. lini aux doses 10000 (A) ou 5000 (B) germes/g de sol. Moyennes de 6 répétitions de 10 plantes suivies de leurs écarts types.

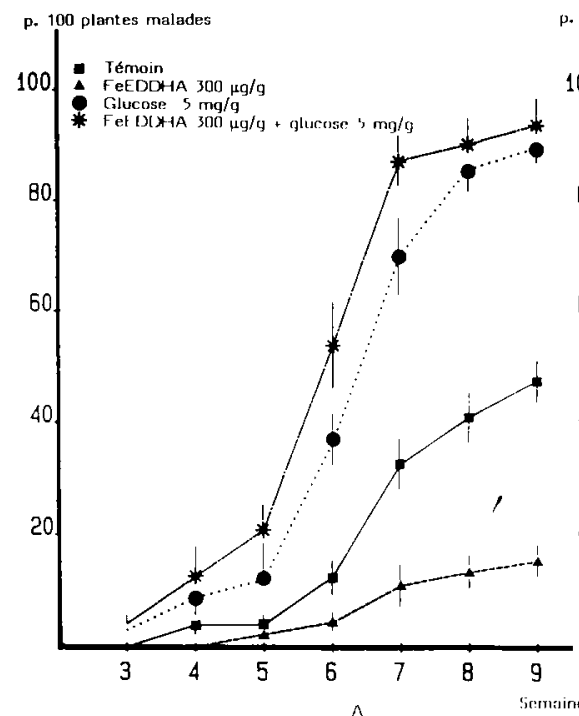

Figure 5

Réceptivité des sols résistant $(A)$ et sensible $(B)$ amendés avec FeED$D H A(300 \mu \mathrm{g} / \mathrm{g})$ et $/$ ou glucose $(A=5 \mathrm{mg} / \mathrm{g}, B=I \mathrm{mg} / \mathrm{g})$ à la fusariose vasculaire du lin : pourcentage de plantes malades après infestation du sol avec F. o. f. sp. lini aux doses 10000 (A) ou 5000 (B) germes/g de sol.

Moyennes de 6 répétitions de 10 plantes suivies de leurs écarts types.

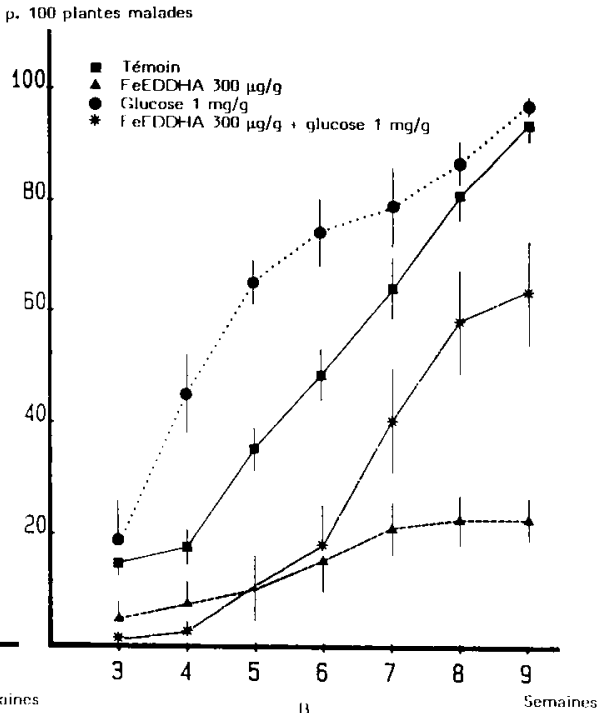

Receptivity to fusarium wilt of flax of the suppressive (A) and con ducive (B) soils supplemented with FeEDDHA $(300 \mu \mathrm{g} / \mathrm{g})$ and $/ \mathrm{or}$ glucose $(A=5 \mathrm{mg} / \mathrm{g}, B=1 \mathrm{mg} / \mathrm{g})$ : percentage of wilted plants after soil infestation with F. o. $f$. sp. lini at $10000(A)$ or 5000 (B) C.F.U.'g of soil.

Means of 6 replications of 10 plants followed by their standard deviations. 


\section{Modification de la réceptivité aux fusarioses vas- culaires par apport de FeEDDHA et de glucose}

Ayant démontré que l'apport du chélate FeEDDHA entraîne une nette diminution du niveau de réceptivité des sols alors que l'apport de glucose, à concentration suffisante, induit une augmentation du niveau de réceptivité, il était intéressant d'étudier les conséquences d'un apport simultané de glucose et de FeEDDHA. Différentes combinaisons de concentration de glucose et de chélate ont été apportées. Mais, afin de simplifier la présentation des résultats, ceux-ci étant parfaitement cohérents, la figure 5 n'indique que ceux obtenus après apport de $300 \mu \mathrm{g} / \mathrm{g}$ FeEDDHA et de 1 ou $5 \mathrm{mg} / \mathrm{g}$ glucose respectivement pour les sols sensible et résistant.

Le niveau de réceptivité des sols amendés avec FeEDDHA ou glucose seuls confirme nos résultats précédents. Ainsi, la gravité de la maladie des sols enrichis avec FeEDDHA est réduite. A l'inverse, l'apport de glucose provoque une augmentation de la gravité de la maladie, toutefois moins nette que lors des essais précédents.

En sol sensible (fig. 5B), l'apport simultané de glucose et de FeEDDHA se traduit pendant 6 semaines par une mortalité identique à celle enregistrée dans le sol amendé avec FeEDDHA seul, c'est-à-dire par une diminution du niveau de réceptivité du sol. Au-delà de la $6^{\mathrm{e}}$ semaine de culture, le pourcentage de plantes malades progresse cependant rapidement.

Dans le sol de Châteaurenard (fig. 5A), l'apport conjoint de glucose et de FeEDDHA provoque une élévation importante du niveau de réceptivité qui atteint même un niveau supérieur à celui induit par le seul apport de glucose à $5 \mathrm{mg} / \mathrm{g}$.

\section{DISCUSSION}

Le premier objectif de ce travail était d'étudier la validité de l'hypothèse concernant le rôle de la disponibilité en fer dans les mécanismes de la résistance des sols aux fusarioses (KLOEPPER et al., 1980 ; SCHER \& BAKER, 1982). Les effets de l'apport de chélates de synthèse dans le sol résistant de Châteaurenard et le sol sensible de Carquefou démontrent que le niveau de réceptivité des sols étudiés varie en fonction de la concentration en fer assimilable par les Fusarium. Globalement ces résultats confirment ceux de SCHER \& BAKER (1982) et montrent que l'apport de ligands, formant avec le fer des complexes stables, diminue la réceptivité des sols alors que l'apport de fer, facilement mobilisable, augmente la réceptivité (fig. 2-3). De plus, ces résultats indiquent que la variation du niveau de réceptivité dépend à la fois de la teneur initiale du sol en fer facilement extractible et de la concentration des chélates apportés. Dans leur ensemble, ces observations sont conformes aux connaissances sur le rôle du fer dans les interactions microbiennes. La constante de stabilité de FeEDDHA étant supérieure à celle de Fe-fusarinine, le ligand EDDHA rend indisponible pour les Fusarium le fer qu'il a chélaté.

Il existe donc une relation entre la concentration de ligand, la quantité de fer immobilisé et le niveau de réceptivité aux fusarioses. Il peut paraître surprenant que le chélate (FeEDDHA) ait le même effet, voire un effet supérieur (fig. 3) à celui du ligand (EDDHA). Cette observation doit être rapprochée de celle de SNEH et al. (1984) qui ont démontré que le chélate FeEDDHA réduit le taux de germination des chlamydospores de Fusarium dans la rhizosphère et non dans le sol lui-même. La plante (LINDSAY, 1974), ainsi que certaines populations de Pseudomonas fluorescents (SCHER et al., 1984) sont capables de prélever l'ion ferrique chélaté par EDDHA. Ce ligand ainsi libéré peut à nouveau chélater l'ion FeIII et ainsi abaisser encore la quantité de fer disponible pour les Fusarium. En présence de plantes, le chélate aurait donc la même efficacité que le ligand.

A l'inverse, EDTA présente une affinité pour le fer inférieure à celle des fusarinines. Les Fusarium peuvent donc utiliser le fer associé à ce ligand, ce qui explique que le niveau de réceptivité des sols aux fusarioses augmente après apport du chélate (FeEDTA). Il est, par contre, difficile de comprendre que le ligand seul ait produit le même effet. Cette observation doit être reliée à la phytotoxicité engendrée par ce ligand, démontrée dans le sol de Carquefou. Cette phytotoxicité déjà établie par d'autres auteurs (VAN DRIEL, 1964 ; CULL, 1981) varie avec le type de sol et elle est nettement plus intense que celle du chélate FeEDTA. Dans le sol de Châteaurenard, la très faible toxicité de FeEDTA ne saurait remettre en cause la conclusion principale de ce travail, à savoir que la disponibilité en fer conditionne la gravité des fusarioses.

Il est intéressant de noter que la compétition pour le fer se manifeste aussi bien dans le sol résistant que dans le sol sensible alors que celui-ci présente des caractéristiques physico-chimiques différentes, en particulier un $\mathrm{pH}$ faible $(5,3)$. Il est établi que le $\mathrm{pH}$ est un des principaux facteurs qui détermine la concentration des sols en fer facilement extractible. Les résultats acquis sont, de ce point de vue, en contradiction avec les indications de BAKER et al. (1986) montrant que la compétition pour le fer cesse de se manifester lorsque le pH des sols est abaissé en dessous de 6,5.

Les travaux présentés ont, par ailleurs, permis de confirmer des résultats antérieurs (ALABOUVETTE, 1983) montrant que le niveau de réceptivité des sols aux fusarioses varie en fonction de la disponibilité en carbone facilement assimilable par les microorganismes. Il est donc permis de conclure que compétition pour l'énergie et compétition pour le fer sont 2 mécanismes qui peuvent se manifester conjointement dans les mêmes sols. Il était donc très intéressant d'observer l'évolution du niveau de réceptivité des sols amendés simultanément avec du glucose et FeEDDHA dont les effets individuels sont opposés.

Dans le sol sensible de Carquefou (fig. 5B), le pourcentage de plantes malades enregistré après apport de glucose $(1 \mathrm{mg} / \mathrm{g})$ et de FeEDDHA $(300 \mu \mathrm{g} / \mathrm{g})$ est pendant 6 semaines identique à celui enregistré après apport du chélate seul. Ceci indique que lorsque le carbone n'est pas un facteur limitant, la gravité de la maladie est déterminée par la disponibilité en fer assimilable par les Fusarium. Au-delà de la $6^{\mathrm{e}}$ semaine de culture, la mortalité augmente rapidement pour devenir identique à celle du témoin. Tout se passe comme si le ligand EDDHA libérait le fer permettant ainsi la manifestation de la maladie. On peut supposer que 
cette molécule organique est dégradée par la microflore tellurique stimulée par l'apport de glucose. Ces résultats sont en concordance avec ceux de SCHER (1986) qui fait état d'une mauvaise stabilité, au cours du temps, de la protection contre la fusariose de l'œillet assurée par FeEDDHA.

Dans le sol de Châteaurenard (fig. 5A), l'apport simultané de FeEDDHA et de glucose provoque une augmentation de la réceptivité du sol supérieure à celle induite par le seul apport de glucose. Il faut admettre que la dégradation de la molécule de chélate libère l'ion FeIII, la maladie se manifeste alors avec intensité, le fer et le carbone n'étant plus limitants.

Lorsque le sol de Châteaurenard est amendé simultanément en glucose et FeEDDHA, le fer ne constitue jamais un facteur limitant. La dégradation de FeEDDHA serait donc plus rapide et plus intense que dans le sol sensible. On peut se demander si, comme pour le glucose (AlABOUVETTE et al., 1985 b) la métabolisation du chélate n'est pas d'autant plus rapide que la biomasse et l'activité microbienne des sols sont élevées. Dans ce cas, la compétition pour le fer, comme la compétition pour l'énergie, serait sous la dépendance de facteurs généraux liés à la richesse globale des sols en microorganismes (COOK \& BAKER, 1983). Il est nécessaire de poursuivre des recherches pour apprécier la validité de cette hypothèse et en tout premier lieu il convient de rechercher et de caractériser les populations microbiennes de ces 2 sols. En effet, l'abondance de certaines populations particulières a été mise en relation avec la résistance des sols. Ainsi, ROUXEL et al. (1979) ont montré que les Fusarium non pathogènes abondants dans le sol de Châteaurenard jouent un rôle indispensable à l'expression de la résistance. SCHER \& BAKER (1982) attribuent aux Pseudomonas fluorescents la résistance des sols de Salinas Valley, ces bactéries productrices de sidérophores, étant selon de nombreux auteurs, cités par LEONG (1986), les principaux responsables de la faible disponibilité en fer dans la rhizosphère des plantes. Il convient de prendre en compte cette hypothèse dans le cas des sols étudiés et pour lesquels il vient d'être montré que le fer peut être un facteur limitant. De nouvelles expérimentations sont donc nécessaires pour synthétiser toutes les informations disponibles et apprécier le rôle respectif de certaines populations de microorganismes et de l'activité microbienne globale.

\section{REMERCIEMENTS}

La concentration des sols en fer a été déterminée au centre de Pédobiologie du C.N.R.S. à Nancy par E. JEANRoY que nous remercions pour sa collaboration.

Reçu le 5 mai 1987. Accepté le ler novembre 1987.

\section{RÉFÉRENCES BIBLIOGRAPHIQUES}

Alabouvette C., 1983. La réceptivité des sols aux fusarioses vasculaires. Rôle de la compétition nutritive entre microorganismes. Thèse Doct. Sci. Nat., Univ. de Nancy, 158 p.

Alabouvette C., 1986. Fusarium-wilt suppressive soils from the Châteaurenard region : review of a 10-year study. Agronomie, 6 (3), 273-284.

Alabouvette C., Couteaudier Y., Louvet J., 1982. Comparaison de la réceptivité de différents sols et substrats de culture aux fusarioses vasculaires. Agronomie, 2 (1), 1-6.

Alabouvette C., Couteaudier Y., Louvet J., 1985a. Recherches sur la résistance des sols aux maladies. XI. Etude comparative du comportement des Fusarium spp. dans un sol résistant et un sol sensible enrichis en glucose. Agronomie, 5 (1), 63-68.

Alabouvette C., Couteaudier Y., Louvet J., 1985b. Recherches sur la résistance des sols aux maladies. XII. Activité respiratoire dans un sol résistant et un sol sensible aux fusarioses vasculaires enrichis en glucose. Agronomie, 5 (1), 69-72.

Atkinson G. F., 1892. Some diseases of cotton. Ala. Auburn agric. Exp. Stn. Bull., 41, 65 p.

Baker R., Elad Y., Sneh B., 1986. Physical, biological and host factors in iron competition in soils. 77-84. In : T. R. SwINBURNE "Iron, siderophores, and plant diseases". NATO Adv. Res. Workshop, U.K. Plenum Press New York and London.

Cook R. J., Baker K. F., 1983. The nature and practice of biological control of plant pathogens. Am. phytopathol. Soc., St Paul MN, USA, $539 \mathrm{p}$.

Cull D. C., 1981. Nutrient film technique : iron nutrition of sweet pepper in relation to root temperature. Acta Hortic., 126, 397-407.

Emery T., 1965. Isolation, characterization, and properties of fusarinine a hydroxamic acid derivative of ornithine. Biochemistry, 4, 1410-1417.

Kloepper J. W., Leong J., Teintze M., Schroth M. N., 1980. Pseudomonas siderophores : a mechanism explaining disease-suppressive soils. Curr. Microbiol., 4, 327-330.
Leong J., 1986. Siderophores : their biochemistry and possible role in the biocontrol of plant pathogens. Ann. Rev. phytopathol., 24, 187-209.

Lindsay W. L., 1974. Role of chelation in micronutrient availability. 507-527. In : E. W. CARSON: "The plant root and its environment'. University Press Virginia, Charlottesville, $691 \mathrm{p}$.

Lockwood J. L., 1977. Fungistasis in soils. Biol. Rev., 52, 1-43.

Louvet J., Rouxel F., Alabouvette C., 1976. Recherches sur la résistance des sols aux maladies. I. Mise en évidence de la nature microbiologique de la résistance d'un sol au développement de la fusariose vasculaire du melon. Ann. Phytopathol, 8 (4), 425-436.

Meyer J. M., Abdallah M. A., 1978. The fluorescent pigment of Pseudomonas fluorescens : biosynthesis, purification and physicochemical properties. J. gen. Microbiol., 107, 319-328.

Neilands J. B., 1973. Microbial iron transport compounds (siderochromes). 167-202. In: G. L. EICHORN : "Inorganic Biochemistry", Elsevier, Amsterdam.

Rouxel F., Alabouvette C., Louvet J., 1979. Recherches sur la résistance des sols aux maladies. IV. Mise en évidence du rôle des Fusarium autochtones dans la résistance d'un sol à la Fusariose vasculaire du Melon. Ann. Phytopathol., 2, 199-207.

Scher F. M., 1986. Biological control of Fusarium wilts by Pseudomonas putida and its enhancement by EDDHA. 109-117. In : T. R. SWINBURNE "Iron, siderophores, and plant diseases". NATO Adv. Res. Workshop, U.K. Plenum Press New York and London.

Scher F. M., Baker R., 1980. Mechanism of biological control in a Fusarium-suppressive soil. Phytopathology, 70, 412-417.

Scher F. M., Baker R., 1982. Effect of Pseudomonas putida and a synthetic iron chelator on induction of soil suppressiveness to Fusarium wilt pathogens. Phytopathology, 72, 1567-1573.

Scher F. M., Dupler M., Baker R., 1984. Effect of a synthetic iron chelate on population densities of Fusarium oxysporum and the biological agent Pseudomonas putida in soil. Can. J. Microbiol., 30, 1271-1275. 
Sneh B., Dupler M., Elad Y., Baker R., 1984. Chlamydospore germination of Fusarium oxysporum f. sp. cucumerinum as affected by fluorescent and lytic bacteria from a Fusarium-suppressive soil. Phytopathology, 74, 1115-1124.

Tello-Marquina J. C., Alabouvette C., Louvet J., 1980. Aptitude à la conservation des microconidies de Fusarium oxysporum f. sp. melonis. Ann. Phytopathol., 12 (3), 227-233.
Toussoun T. A., 1975. Fusarium-suppressive soils, 145-151. In : G. W. BRUEHL: "Biology and control of soil-borne plant pathogens". Am. Phytopathol. Soc., 217 p.

Van Driel W., 1964. The effect of iron EDTA on the growth and metabolism of tomato plants in water culture. Plant Soil, 20, $85-104$. 